Article

\title{
Detecting Suspicious Texts Using Machine Learning Techniques
}

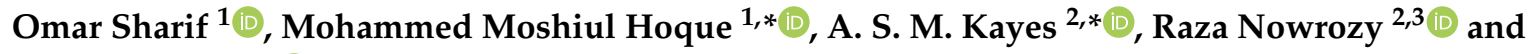 \\ Iqbal H. Sarker ${ }^{1}$ (D) \\ 1 Department of Computer Science and Engineering, Chittagong University of Engineering and Technology, \\ Chittagong 4349, Bangladesh; omar.sharif@cuet.ac.bd (O.S.); iqbal@cuet.ac.bd (I.H.S.) \\ 2 Department of Computer Science and Information Technology, La Trobe University, Plenty Road, Bundoora, \\ VIC 3086, Australia; r.nowrozy@latrobe.edu.au \\ 3 College of Engineering and Science, Victoria University, Ballarat Road, Footscray, VIC 3011, Australia \\ * Correspondence: moshiul_240@cuet.ac.bd (M.M.H.); a.kayes@latrobe.edu.au (A.S.M.K.)
}

Received: 5 August 2020; Accepted: 12 September 2020; Published: 18 September 2020

\begin{abstract}
Due to the substantial growth of internet users and its spontaneous access via electronic devices, the amount of electronic contents has been growing enormously in recent years through instant messaging, social networking posts, blogs, online portals and other digital platforms. Unfortunately, the misapplication of technologies has increased with this rapid growth of online content, which leads to the rise in suspicious activities. People misuse the web media to disseminate malicious activity, perform the illegal movement, abuse other people, and publicize suspicious contents on the web. The suspicious contents usually available in the form of text, audio, or video, whereas text contents have been used in most of the cases to perform suspicious activities. Thus, one of the most challenging issues for NLP researchers is to develop a system that can identify suspicious text efficiently from the specific contents. In this paper, a Machine Learning (ML)-based classification model is proposed (hereafter called STD) to classify Bengali text into non-suspicious and suspicious categories based on its original contents. A set of ML classifiers with various features has been used on our developed corpus, consisting of 7000 Bengali text documents where 5600 documents used for training and 1400 documents used for testing. The performance of the proposed system is compared with the human baseline and existing ML techniques. The SGD classifier 'tf-idf' with the combination of unigram and bigram features are used to achieve the highest accuracy of $84.57 \%$.
\end{abstract}

Keywords: natural language processing; suspicious text detection; Bengali language processing; machine learning; text classification; feature extraction; suspicious corpora

\section{Introduction}

Due to the effortless access of the Internet, world wide web, blogs, social media, discussion forums, and online platforms via digital gadgets have been producing a massive volume of digital text contents in recent years. It is observed that all the contents are not genuine or authentic; instead, some contents are faked, fabricated, forged, or even suspicious. It is very unpropitious with this rapid growth of digital contents that the ill-usage of the Internet has also been multiplied which governs the boost in suspicious activities [1]. Suspicious contents are increasing day by day because of ill-usage of the Internet by a few individuals to promulgate fierceness, share illegal activities, bullying other people, perform smishing, publicize incitement related contents, spread fake news, and so on. According to the FBI's Internet Crime Complaint Center (IC3) report, a total of 467,361 complaints received in the year 2019 related to internet-facilitated criminal activity [2]. Moreover, several extremist users use 
social media or blogs to spread suspicious and violent contents which can be considered one kind of threat to national security [3].

Around 245 million people are speaking in Bengali as their native tongue, which makes it the 7th most spoken language in the world [4]. However, research on Bengali Language Processing (BLP) is currently in its initial stage, and there are no significant amount of works that have been conducted yet like English, Arabic, Chinese, or other European languages that make Bengali a resource constrained language [5]. As far as we are concerned, there has been no research conducted up to now on suspicious text detection in the Bengali language. However, such systems are required to ensure the security as well as mitigate national threats in cyber-space.

Suspicious contents are those contents that hurt religious feelings, provoke people against government and law enforcement agencies, motivate people to perform acts of terrorism, perform criminal acts by phishing, smishing, and pharming, instigate a community without any reason, and execute extortion acts [6-9]. As examples, social media has already used as a medium of communication in Boston attack and the revolution in Egypt [10]. The suspicious contents can be available in the form of video, audio, images, graphics, and text. However, text plays an essential role in this context as it is the most widely used medium of communication in cyber-space. Moreover, the semantic meaning of a conversation can be retrieved by analyzing text contents which is difficult in other forms of content. In this work, we focus on analyzing text content and classifying the content into suspicious or non-suspicious.

A text could be detected as suspicious if it contained suspicious contents. It is impossible to detect suspicious texts from the enormous amount of internet text contents manually [11]. Therefore, the automatic detection of suspicious text contents should be developed. Responsible agencies have been demanding some smart tool/system that can detect suspicious text automatically. It will also be helpful to identify potential threats in the cyber-world which are communicated by text contents. Automatic detection of suspicious text system can easily and promptly detect the fishy or threatening texts. Law and enforcement authority can take appropriate measures immediately, which in turn helps to reduce virtual harassment, and suspicious and criminal activities mediated through online. However, it is a quite challenging task to classify the Bengali text contents into suspicious or non-suspicious class due to its complex morphological structure, enormous numbers of synonym, and rich variations of verb auxiliary with the subject, person, tense, aspect, and gender. Moreover, scarcity of resources and lack of benchmark Bengali text dataset are the major barriers to build a suspicious text detection system and make it more difficult to implement compared to other languages. Therefore, the research question addressing in this paper is- "RQ: How can we effectively classify potential Bengali texts into suspicious and non-suspicious categories?"

To address this research question in this work, we first develop a dataset of suspicious and non-suspicious texts considering a number of well-known Bengali data sources, such as Facebook posts, blogs, websites, and newspapers. In order to process the textual data, we take into account unigram, bigram, trigram features using tf-idf and a bag of words feature extraction technique. Once the feature extraction has been done, we employ the most popular machine learning classifiers (i.e., logistic regression, naive Bayes, random forest, decision tree, and stochastic gradient descent) to classify whether a given text is suspicious or not. We have also performed a comparative analysis of these machine learning models utilizing our collected datasets. The key contributions of our work are illustrated in the following:

- Develop a corpus containing 7000 text documents labelled as suspicious or non-suspicious.

- Design a classifier model to classify Bengali text documents into suspicious or non-suspicious categories on developed corpus by exploring different feature combination.

- Compare the performance of the proposed classifier with various machine learning techniques as well as the existing method.

- Analyze the performance of the proposed classifier on different distributions of the developed dataset. 
- Exhibits a performance comparison between human expert (i.e., baseline) and machine learning algorithms.

We expect that the work presented in this paper will play a pioneering role in the development of Bengali suspicious text detection systems. The rest of the paper organized as follows: Section 2 presents related work. In Section 3, a brief description of the development of suspicious Bengali corpus and its several properties have explained. Section 4 explained the proposed Bengali suspicious text document classification system and its significant constituents. Section 5 described the evaluation techniques used to assess the performance of the proposed approach. Results of the experiments are also presented in this section. Finally, in Section 6, we concluded the paper with a summary and discussed the future scopes.

\section{Related Work}

Suspicious contents detection is a well-studied research issue for the highly resourced languages like Arabic, Chinese, English, and other European languages. However, no meaningful research activities have been conducted yet to classify text with suspicious content in the BLP domain. A machine learning-based system developed to detect promotion of terrorism by analyzing the contents of a text. Iskandar et al. [12] have collected data from Facebook, Twitter, and numerous micro-blogging sites to train the model. By performing a critical analysis of different algorithms, they showed that Naïve Bayes is best suited for their work as it deals with probabilities [13]. Johnston et al. [14] proposed a neural network-based system which can classify propaganda related to the Sunni (Sunni is a class of Islamic believer group of Muslims: www.britannica.com/topic/Sunni) extremist users on social media platforms. Their approach obtained $69.9 \%$ accuracy on the developed dataset. A method to identify suspicious profiles within social media presented where normalized compression distance was utilized to analyze text [15]. Jiang et al. [16] discusses current trends and provides future direction to determine suspicious behaviour in various mediums of communications. The researchers investigated the novelty of true and false news on 126,000 stories that tweeted 4.5 million times using ML techniques [17]. An automated system explained the technique of detecting hate speech from the Twitter data [18]. Logistic regression with regularization outperforms other algorithms by attaining the accuracy of $90 \%$. An intelligent system introduced to detect suspicious messages from Arabic tweets [19]. This system yields maximum accuracy of $86.72 \%$ using SVM with a limited number of data and class. Dinakar et al. [20] developed a corpus of YouTube comments for detecting textual cyberbullying using a multiclass and binary classifier. A novel approach presented of detecting Indonesian hate speech by using SVM, lexical, word unigram and tf-idf features [21]. A method described to detect abusive content and cyberbullying from Chinese social media. Their model achieved 95\% accuracy by using LSTM and taking characteristic and behavioural features of a user [22]. Hammer [23] discussed a way of detecting violence and threat from online discussions towards minority groups. This work considered the manually annotated sentences with bigram features of essential words.

Since Bengali is an under-resourced language, the amount of digitized text (related to suspicious, fake, or instigation) is quite less. In addition to that, no benchmark dataset is available on the suspicious text. Due to these reasons, very few research activities have carried out in this area of BLP, which are mainly related to hate, threat, fake and abusive text detection. Ishmam et al. [24] compare machine learning and deep learning-based model to detect hateful Bengali language. Their method achieved $70.10 \%$ accuracy by employing a gated recurrent neural network (GRNN) method on a dataset of six classes and $5 \mathrm{~K}$ documents collected from numerous Facebook pages. The reason behind this poor accuracy is the less number of training documents in each class (approximately 900). Most importantly, they did not define the classes clearly, which is very crucial for the hateful text classification task. Recent work explained a different machine and deep learning technique to detect abusive Bengali comments [25]. The model acquired $82 \%$ accuracy by using RNN on 4700 Bengali text documents. Ehsan et al. [26] discussed another approach of detecting abusive Bengali text by combining different 
n-gram features and ML techniques. Their method obtained the highest accuracy for SVM with trigram features. A method to identify malicious contents from Bengali text is presented by Islam et al. [27]. This method achieved $82.44 \%$ accuracy on an unbalanced dataset of 1965 instances by applying the Naive Bayes algorithm. Hossain et al. [28] develop a dataset of $50 \mathrm{k}$ instances to detect fake news in Bangla. They have extensively analyzed linguistic as well as machine learning-based features. A system demonstrated the technique to identify the threats and abusive Bengali words in social media using SVM with linear kernel [29]. The model experimented with 5644 text documents and obtained the maximum accuracy of $78 \%$.

As far as we aware, none of the remarkable research conveyed so far that focuses on detecting suspicious Bengali text. Our previous approach used logistic regression with BoW features extraction technique to detect suspicious Bengali text contents [30]. However, that work considered only 2000 text documents and achieved an accuracy of $92 \%$. In this work, our main concern is to develop the ML-based suspicious Bengali text detection model trained on our new dataset by exploring various n-gram features and feature extraction techniques.

\section{A Novel Suspicious Bangla Text Dataset}

Up until this date, no dataset is available for identifying Suspicious Bengali Texts (SBT). Therefore, we developed a Suspicious Bengali Text Dataset (SBTD), which is a novel annotated corpus to serve our purpose. The following subsection explains the definition of SBT with its inherent characteristics and details statistics of the developed SBTD.

\subsection{Suspicious Text and Suspicious Text Detection}

Suspicious Text Detection (STD) system classifies a text $t_{i} \in T$ from a set of texts $T=\left\{t_{1}, t_{2}, \ldots, t_{m}\right\}$ into a class $c_{i} \epsilon C$ from a set of two classes $C=\left\{C_{s}, C_{n s}\right\}$. The task of STD is to automatically assign $t_{i}$ to $c_{i}:\left\langle t_{i}, c_{i}\right\rangle$.

Deciding whether a Bengali text is suspicious or not is not so simple even for language experts because of its complicated morphological structure, rich variation in sentence formation, and lack of defining related terminology. Therefore, it is very crucial to have a clear definition of SBT for making the task of STD smoother. In order to introduce a reasonable definition concerning the Bengali language, several definitions of violence, incitement, suspicious, and hatred contents have analyzed. Most of the information collected from the different social networking websites and scientific papers summarized in Table 1.

Table 1. Definitions of hatred, incitement and violent contents according to different social networking websites, organization, and scientific studies

\begin{tabular}{ll}
\hline Source & Definition \\
\hline Facebook & $\begin{array}{l}\text { "Contents that incite or facilitate serious violence pose credible threat to the } \\
\text { public or personal safety, instructions to make weapons that could injure or } \\
\text { kill people and threats that lead to physical harm towards private individuals } \\
\text { or public figures" [6]. }\end{array}$ \\
\hline Twitter & $\begin{array}{l}\text { “One may not promote terrorism or violent extremism, harasses or threaten } \\
\text { other people, incite fury toward a particular or a class of people" [31]. }\end{array}$ \\
\hline YouTube & $\begin{array}{l}\text { “Contents that incite others to promote or commit violence against } \\
\text { individuals and groups based on religion, nationality, ethnicity, sex/gender, } \\
\text { age, race, disability, gender identity/sexual orientation" [32]. }\end{array}$ \\
\hline Council of Europe (COE) & $\begin{array}{l}\text { "Expression which incite, spread, promote or justify violence toward a } \\
\text { specific individual or class of persons for a variety of reasons" [33]. }\end{array}$ \\
\hline Paula et al. & $\begin{array}{l}\text { "Language that glorify violence and hate, incite people against groups based } \\
\text { on religion, ethnic or national origin, physical appearance, gender identity } \\
\text { or other" [7]. }\end{array}$ \\
\hline
\end{tabular}


The majority of the quoted definitions focus on similar attributes such as incitement of violence, promotion of hate and terrorism, and threatening a person or group of people. These definitions cover the larger aspect of suspicious content from video, text, image, cartoon, illustrations and graphics. Nevertheless, in this work, we concentrate on detecting suspicious content form the text contents only. Analyzing the contents and properties of these definitions guided us to present a definition of suspicious Bengali text as follows:

"Suspicious Bengali texts are those texts which incite violence, encourage in terrorism, promote violent extremism, instigate political parties, excite people against a person or community based on some specific characteristics such as religious beliefs, minority, sexual orientation, race and physical disability."

\subsection{Development of SBT Corpora}

Bengali is the resource-constrained language due to its scarcity of digitized text contents and unavailability of benchmark datasets. By considering the explanation of SBT and the characteristics of suspect activity defined by the U. S. department of homeland security, we accumulated the text data from various online sources [34]. We endorsed the same technique of developing datasets, as explained by Das et al. [35]. Figure 1 illustrates the process of dataset development.

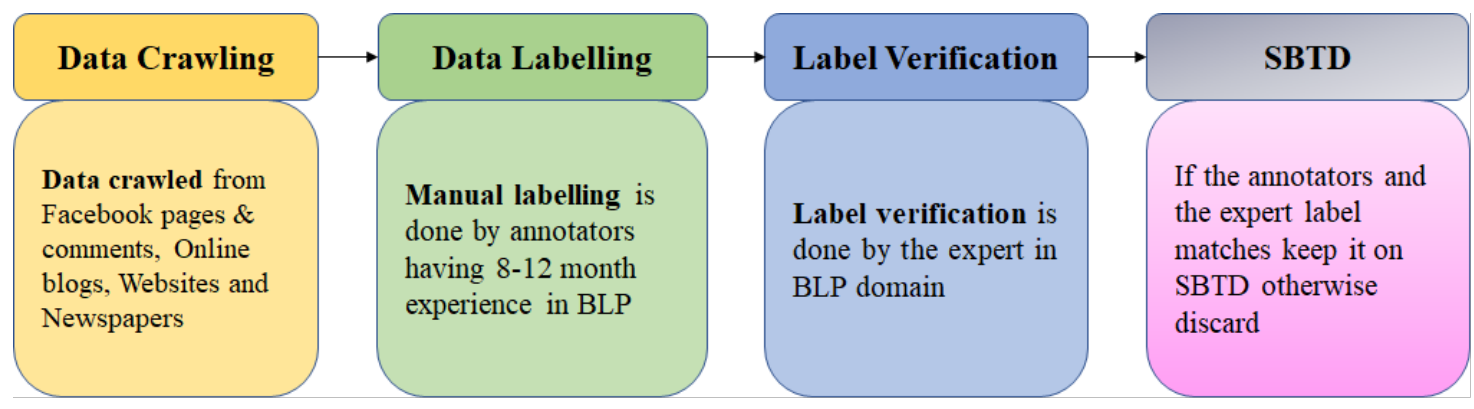

Figure 1. Process of dataset accumulation.

Data crowd-sourcing: Figure 2 shows the total number of texts collected from different sources in terms of suspicious (S) and non-suspicious (NS) classes. We have crawled a total of 7115 texts among them 3557 texts are S, and 3558 texts are NS. In the case of the suspicious class, $12.2 \%$ of source texts collected from the website (W), 12\% data collected from the Facebook comment (FC), and $10.2 \%$ from the newspaper $(\mathrm{N})$. Other sources such as Facebook posts $(\mathrm{FP})$ and online blogs $(\mathrm{OB})$ contributed $8.9 \%$ and $5.4 \%$ of text data. On the other hand, a significant portion of non-suspicious source texts collected from the newspapers (30.4\%). A total of $7.8 \%$ of non-suspicious texts were collected from the OB, $5.6 \%$ from the $\mathrm{W}$ and $3.2 \%$ from the FC. A tiny portion of the texts was accumulated from various sources (such as novels and articles) in both classes. As the sources of the newspapers, the three most popular Bangladeshi newspapers are considered (such as the daily Jugantor, the daily Kaler Kontho, and the daily Prothom Alo) for accumulating the texts.

Data labelling: Crowd-sourced data are initially labelled by five undergraduate students of Chittagong University of Engineering and Technology who have 8-12 months of experience in the BLP domain. They are also doing their undergraduate thesis on BLP and attended several seminars, webinars, and workshops on computational linguistics and NLP.

Label verification: The expert verifies the data labels. A professor or a PhD student having more than five years of experience or any researcher having vast experience in the BLP domain can be considered as an expert. The final labels $\left(C_{n s}, C_{s}\right)$ of data are decided by pursuing the process described in Algorithm 1. 


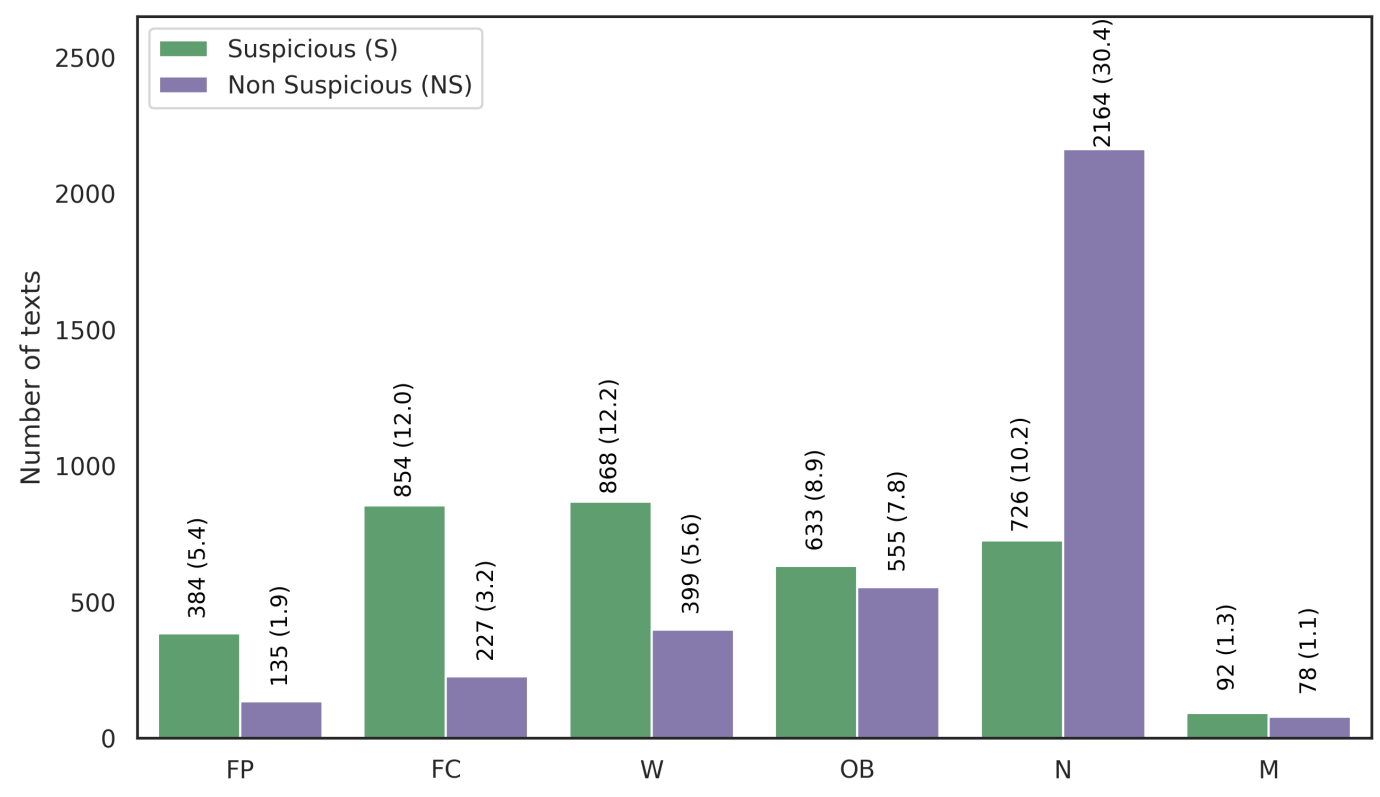

Figure 2. Source texts distribution in suspicious (S) and non-suspicious (NS) categories. The acronyms FP, FC, W, OB, N, and M denote Facebook pages, Facebook comments, websites, online blogs, newspapers, and miscellaneous, respectively.

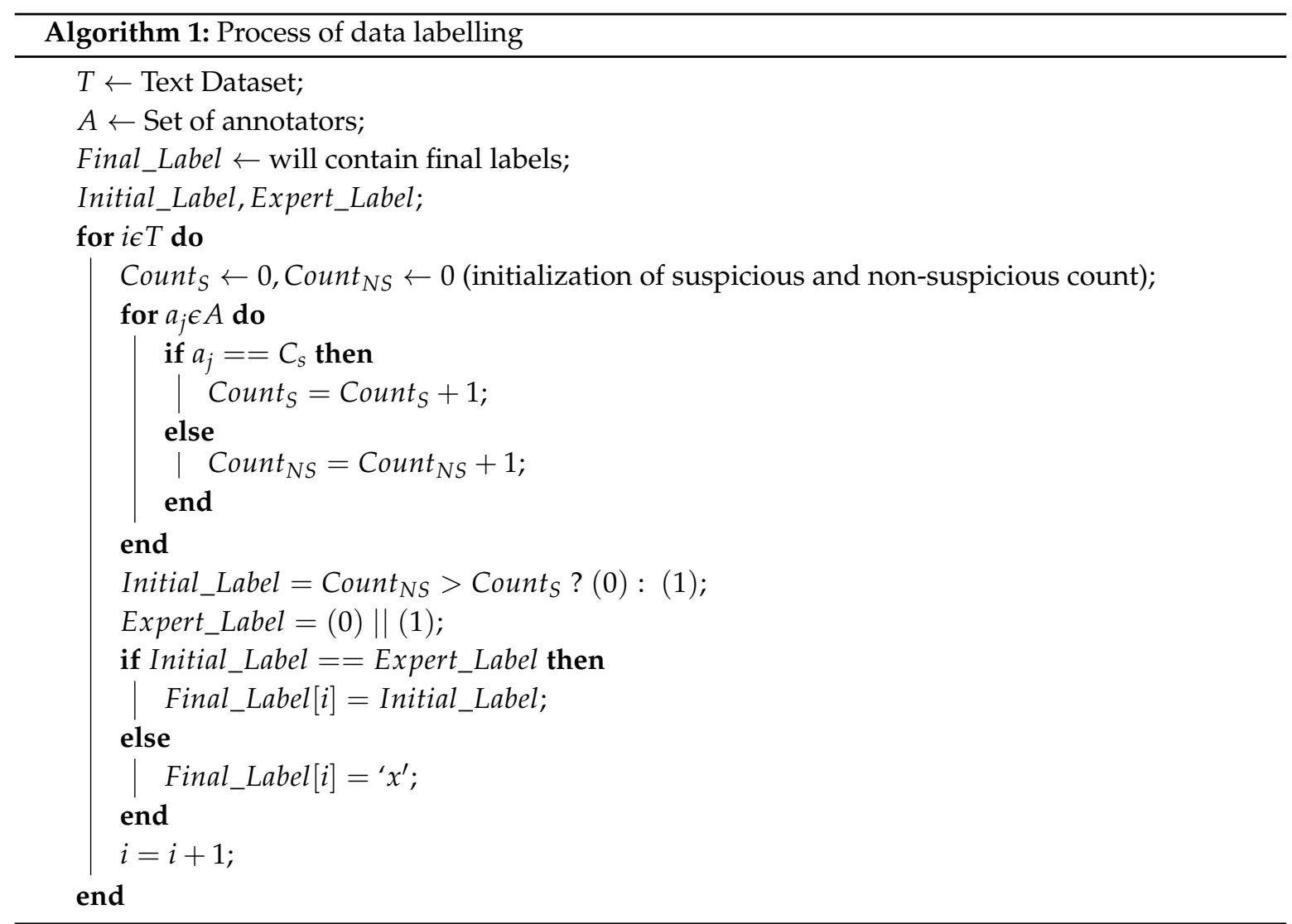

For each text in $T$, the annotator labels are counted. $a_{j}$ indicates the $j^{\text {th }}$ annotator label for the $i$ th text. If the annotator label is suspicious $\left(C_{S}\right)$, then suspicious count $\left(\right.$ Count $\left._{S}\right)$ will be increased; otherwise, the non-suspicious count $\left(\mathrm{Count}_{N S}\right)$ will increase. Majority voting [36] will decide the initial label. If the non-suspicious count is greater than the suspicious count, then the initial label will be non-suspicious (0); otherwise, suspicious (1). After that, the expert will label the text as either 
non-suspicious or suspicious. If the initial label matches the expert label, then it will be the final label. When disagreement increased, the label marked with ' $x$ ', and the final label will be decided by a discussion between the experts and the annotators. If they agree on a label, it will be added to SBTD; otherwise, it will be discarded. It is noted that most of the disagreement was aroused for data of the suspicious class. Among 900 disagreements, only $5-7 \%$ disagreement occurs for non-suspicious classes. A small number of labels and their corresponding texts discarded from the crawled dataset due to the disagreement between experts and annotators. Precisely, 57 for the suspicious class and 58 for the non-suspicious class. We got $9.57 \%$ deviation on the agreement among annotators for suspicious class and $2.34 \%$ deviation for the non-suspicious class. This deviation is calculated by averaging pairwise deviation between annotators. Cohen's kappa [37] between human expert and initial annotators are $88.6 \%$, which indicates a high degree of similarity between them. Table 2 shows a sample data of our corpus. Our data are stored in the corpus in Bangla form, but Banglish form and the English translation is given here for better understanding.

Table 2. A sample text with corresponding metadata on SBTD.

\begin{tabular}{cl}
\hline Domain & https://www.prothomalo.com/ \\
Source & Newspaper \\
Crawling Date & 18 January 2019 \\
& (Banglish form: "BPL a ek durdanto match gelo. Khulna Titans ke \\
& tin wicket a hariye dilo comilla victoria"). (English form: "A great \\
Text $\left(\boldsymbol{t}_{\boldsymbol{i}}\right)$ & match was played in BPL. Comilla Victoria defeated Khulna Titans \\
& by 3 wickets") \\
Final Label & 0 (Non-Suspicious) \\
\hline
\end{tabular}

Table 3 summarizes the several properties of the developed dataset. Creating SBTD was the most challenging task for our work because all the texts demanded manual annotation. It took around ten months of relentless work to build this SBTD. Some metadata have also been collected with the text.

Table 3. Statistics of the dataset.

\begin{tabular}{ccc}
\hline Attributes & Suspicious $\left(C_{s}\right)$ & Non-Suspicious $\left(C_{n s}\right)$ \\
\hline Number of documents & 3500 & 3500 \\
Total words & 95,629 & 252,443 \\
Total unique words & 18,236 & 36,331 \\
Avg. number of words & 27.32 & 72.12 \\
Maximum text length & 427 & 2102 \\
Minimum text length & 3 & 5 \\
Size (in bytes) & 688,128 & 727,040 \\
\hline
\end{tabular}

\section{Proposed System}

The primary objective of this work is to develop a machine learning-based system that can identify suspicious content in Bengali text documents. Figure 3 shows a schematic process of the proposed system that is comprised of four major parts: preprocessing, feature extraction, training and prediction. Input texts are processed by following several preprocessing steps explained in Section 4.1. Feature extraction methods are employed on the processed texts to extract features. In the training phase, exploited features are used to train the machine learning classifiers (i.e., Stochastic gradient descent, Logistic regression, Decision tree, Random forest, and Multinomial Naïve Bayes). Finally, the trained model will be used for classification in the prediction step. The following subsections include the detailed explanation of the significant parts of the proposed system. 


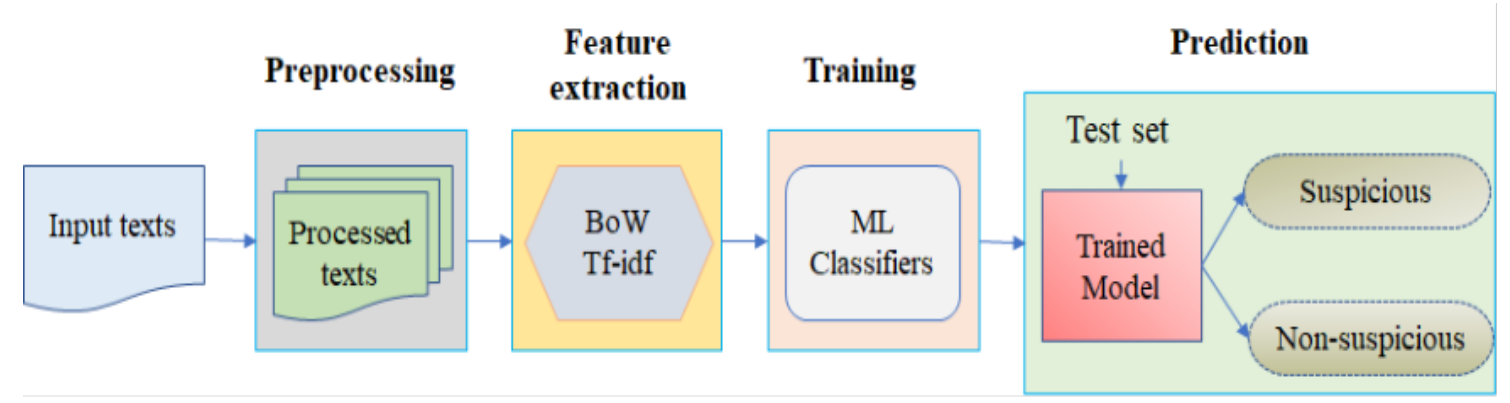

Figure 3. Schematic process of the proposed suspicious text detection system.

\subsection{Preprocessing}

Preprocessing is used to transform raw data into an understandable form by removing inconsistencies and errors. Suppose that a Bengali text document $t_{i}=($ Banglish form $)$ "Ei khulna titans ke, tin wickete hariye dilo comilla victoria, ?...।" (English translation: Comilla Victoria defeated this Khulna Titans by three wickets.) of the dataset $T[]$ can be preprocessed according to the following steps:

- Redundant characters removal: Special characters, punctuation, and numbers are removed from each text $t_{i}$ of the dataset $T[]$. After this, $t_{i}$ becomes "Ei khulna titans ke tin wickete hariye dilo comilla victoria".

- Tokenization: Each text document $t_{i}$ is detruncated into its constituent words. A word vector of dimension $\mathrm{k}$ is obtained by tokenizing a text, $t_{i}$ having $\mathrm{k}$ words, where $t_{i}=w^{<1>}, w^{<2>}, \ldots, w^{<k>}$. Tokenization gives a list of words of the input text such as $t_{i}=\left[{ }^{\prime} \mathrm{Ei}^{\prime}\right.$, 'khulna', 'titans', 'ke', 'tin', 'wickete', 'hariye', 'dilo', 'comilla', 'victoria']

- Removal of stop words: Words that have no contribution in deciding whether a text $t_{i}$ is $\left(C_{s}\right)$ or $\left(C_{n s}\right)$ is considered as unnecessary. Such words are dispelled from the document by matching with a list of stop words. Finally, after removing the stop words, the processed text as, $t_{i}=$ "Khulna titans ke tin wickete hariye dilo comilla victoria". (English translation: Comilla Victoria defeated Khulna Titans by three wickets) will be used for training.

With the help of the above operations, a set of processed texts is created. These texts are stored chronologically in a dictionary in the form of array indexing $A\left[t_{1}\right] \ldots A\left[t_{7000}\right]$ with a numeric $(0,1)$ label. Here, 0 and 1 represent non-suspicious and suspicious class, respectively.

\subsection{Feature Extraction}

Machine learning models could not possibly learn from the texts that we have prepared. Feature extraction performs numeric mapping on these texts to find some meaning. This work explored the bag of words (BoW) and term frequency-inverse document frequency (tf-idf) feature extraction techniques to extract features from the texts.

The BoW technique uses the word frequencies as features. Here, each cell gives the count $(c)$ of a feature word $\left(f_{w i}\right)$ in a text document $\left(t_{i}\right)$. Unwanted words may get higher weights than the context-related words on this technique. The Tf-idf technique [38] tries to mitigate this weighting problem by calculating the tf-idf value according to Equation (1):

$$
t f-i d f\left(f_{w i}, t_{i}\right)=t f\left(f_{w i}, t_{i}\right) \log \frac{m}{\left|t \epsilon m: f_{w} \epsilon t\right|}
$$

Here, $t f-i d f\left(f_{w i}, t_{i}\right)$ indicates the tf-idf value of word $f_{w i}$ in text document $\left(t_{i}\right), t f\left(f_{w i}, t_{i}\right)$ indicates the frequency of word $f_{w i}$ in text document $\left(t_{i}\right), m$ means total number of text documents, and $\left|t \epsilon m: f_{w} \in t\right|$ represents the number of text document $t$ containing word $f_{w}$.

Tf-idf value of the feature words $\left(\left(f_{w}\right)\right)$ puts more emphasis on the words related to the context than other words. To find the final weighted representation of the sentences, compute the Euclidean 
norm after calculating $t f-i d f$ value of the feature words of a sentence. This normalization set high weight on the feature words with smaller variance. Equation (2) computes the norm:

$$
X_{\text {norm }}(i)=X_{i} / \sqrt{\left(X_{1}\right)^{2}+\left(X_{2}\right)^{2}+\ldots+\left(X_{n}\right)^{2}}
$$

Here, $X_{n o r m}(i)$ is the normalized value for the feature word $f_{w i}$ and $X_{1}, X_{2}, \ldots, X_{n}$ are the $t f-i d f$ value of the feature word $f_{w 1}, f_{w 2}, \ldots, f_{w n}$, respectively. Features picked out by both techniques have been applied on the classifier.

BoW and tf-idf feature extraction techniques are used to extract the features. Table 4 presents the sample feature values for first five feature words $\left(f_{w 1}, f_{w 2}, f_{w 3}, f_{w 4}, f_{w 5}\right)$ of the first four text samples $\left(t_{1}, t_{2}, t_{3}, t_{4}\right)$ in our dataset. Features exhibited by an array of size $(m * n)$ having $m$ rows and $n$ columns. A total of 7000 text documents $t_{1}, t_{2}, \ldots, t_{7000}$ are represented in rows while all the feature words $f_{w 1}, f_{w 2}, \ldots, f_{3000}$ are represented in columns. In order to reduce the complexity and computational cost, 3000 most frequent words considered as the feature words among thousands of unique words.

Table 4. Small fragment of extracted feature values for the first four texts of the dataset.

\begin{tabular}{|c|c|c|c|c|c|c|}
\hline & Technique & $f_{w 1}$ & $f_{w 2}$ & $f_{w 3}$ & $f_{w 4}$ & $f_{w 5}$ \\
\hline & & \multicolumn{5}{|c|}{ Sample Feature Values } \\
\hline \multirow[b]{2}{*}{$t_{1}$} & BoW & 1 & 0 & 4 & 6 & 2 \\
\hline & tf-idf & 0.35 & 0.03 & 0.42 & 0.59 & 0.23 \\
\hline \multirow{2}{*}{$t_{2}$} & BoW & 5 & 2 & 1 & 8 & 10 \\
\hline & tf-idf & 0.47 & 0.28 & 0.11 & 0.65 & 0.72 \\
\hline \multirow[b]{2}{*}{$t_{3}$} & BoW & 0 & 1 & 3 & 12 & 5 \\
\hline & tf-idf & 0.04 & 0.11 & 0.22 & 0.75 & 0.44 \\
\hline \multirow{2}{*}{$t_{4}$} & BoW & 2 & 0 & 7 & 4 & 9 \\
\hline & tf-idf & 0.17 & 0.02 & 0.62 & 0.48 & 0.65 \\
\hline
\end{tabular}

The model extracted linguistic n-gram features of the texts. The N-gram approach is used to take into account the sequence order in a sentence in order to make more sense from the sentences [39]. Here, ' $n$ ' indicates the number of consecutive words that can be treated as one gram. N-gram, as well as a combination of n-gram features, will be applied in the proposed model.

Table 5 shows the illustration of various n-gram features. The combination of two feature extraction techniques and n-gram features will be applied to find the best-suited model for the accomplishment of suspicious Bengali text detection.

Table 5. Representation of different $\mathrm{N}$-gram features for a sample Bangla text (Banglish form).

\begin{tabular}{c} 
N-grams $\begin{array}{c}\text { 'Khulna titans ke tin wickete hariye dilo comilla victoria"' } \\
\text { unigrams }\end{array}$ \\
$\begin{array}{r}\text { 'khulna', 'titans', 'ke', 'tin', 'wickete', 'hariye', 'dilo', 'comilla', 'victoria' } \\
\text { 'khulna titans', 'titans ke', 'ke tin', 'tin wickete', 'wickete hariye', 'hariye } \\
\text { dilo', 'dilo comilla', 'comilla victoria' }\end{array}$ \\
$\begin{array}{r}\text { trigrams } \\
\text { 'khulna titans ke', 'titans ke tin', 'ke tin wickete', 'tin wickete hariye', } \\
\text { 'wickete hariye dilo', 'hariy dilo comilla', 'dilo comilla victoria' }\end{array}$ \\
\hline
\end{tabular}

\subsection{Training}

Features that we obtained from the previous step were used to train the machine learning model by employing different popular classification algorithms [40]. These algorithms are stochastic gradient descent (SGD), logistic regression (LR), decision tree (DT), random forest (RF), and multinomial naïve Bayes (MNB). We analyze these algorithms and explain their structure in our system in the following subsections. 


\subsubsection{Stochastic Gradient Descent}

Stochastic gradient descent (SGD) is a well-known technique used to solve ML problems [41]. It is an optimization technique where a sample is selected randomly in each iteration instead of whole data samples. Equations (3) and (4) represent the weight update process for gradient descent and stochastic gradient descent at the $j^{\text {th }}$ iteration:

$$
\begin{aligned}
& w_{j}:=w_{j}-\alpha \frac{\partial J}{\partial w_{j}} \\
& w_{j}:=w_{j}-\alpha \frac{\partial J_{i}}{\partial w_{j}}
\end{aligned}
$$

Here, $\alpha$ indicates the learning rate, $J$ represents the cost over all training examples, and $J_{i}$ is the cost of the ith training example. It is computationally costly to calculate the sum of the gradient of the cost function of all the samples; thus, each iteration takes a lot of time to complete [42]. To address this issue, SGD takes one sample randomly in each iteration and calculate the gradient. Although it takes more iteration to converge, it can reach the global minima with shorter training time. Algorithm 2 explains the process of SGD. $C$ is the optimizer that takes $\theta$ and returns the cost and gradient. $\alpha$ and theta0 represents the learning rate and the starting point of SGD, respectively.

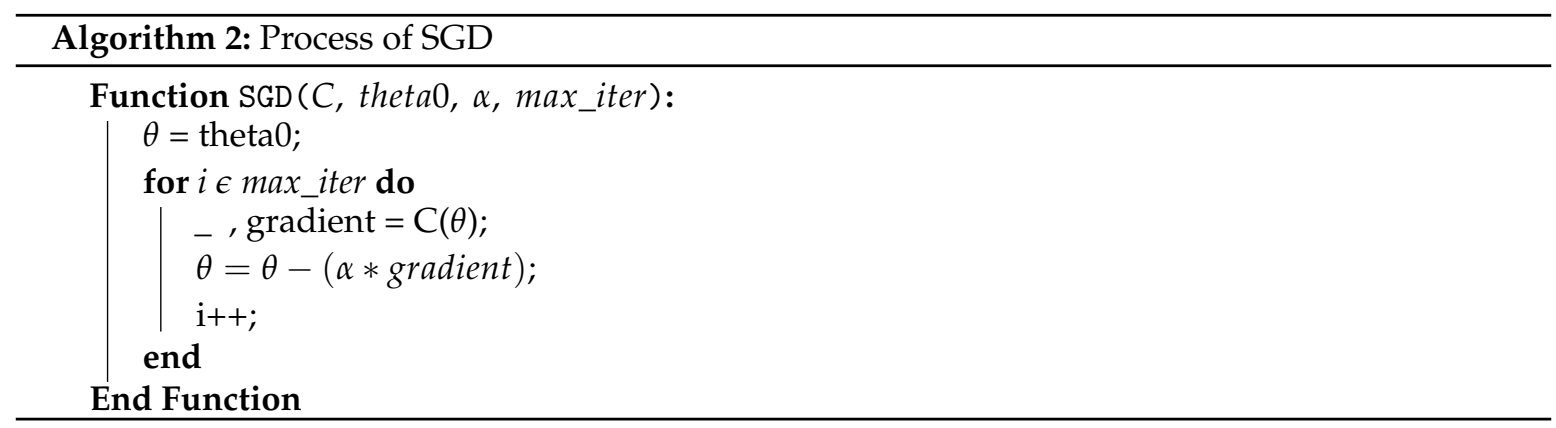

We implemented the SGD classifier with ' $\log$ ' loss function and the ' 12 ' regularization technique. We choose a maximum number of iterations on a trial and error basis. Finally, 40 iterations are used and samples are randomly shuffled during training.

\subsubsection{Logistic Regression}

Logistic regression [43] is well suited for the binary classification problem. Equations (5)-(6) define the logistic function that determines the output of logistic regression:

$$
h_{\theta}(x)=\frac{1}{1+\exp \left(-\theta^{T} x\right)}
$$

Cost function is,

$$
\begin{gathered}
C(\theta)=\frac{1}{m} \sum_{i-1}^{m} c\left(h_{\theta}\left(x^{i}\right), y^{i}\right) \\
c\left(h_{\theta}(x), y\right)= \begin{cases}-\log \left(1-h_{\theta}(x)\right) & \text { if } y=0 \\
-\log \left(h_{\theta}(x)\right) & \text { if } y=1\end{cases}
\end{gathered}
$$

Here, $m$ indicates the number of training examples, $h_{\theta}\left(x^{i}\right)$ presents the hypothesis function of the ith training example, and $y^{i}$ is the input label of $i$ th training example. We used the ' 12 ' norm to penalize the classifier, and the 'lbfgs' optimizer is used for a maximum of 100 iterations. The default value of the inverse of regularization strength is used with a random state 0 . 


\subsubsection{Decision Tree}

The decision tree has two types of nodes: external and internal. External nodes represent the decision class while internal nodes have the features essential for making classification [44]. The decision tree was evaluated in the top-down approach where homogeneous data were partitioned into subsets. Its entropy determines the homogeneity of samples, which is calculated by the Equation (7):

$$
E(S)=\sum_{l=1}^{n} p_{i} \log _{2} p_{i}
$$

Here, $p_{i}$ is the probability of a sample in the training class, and $E(S)$ indicates entropy of the sample. We used entropy to determine the quality of the split. All of the features considered during the split to choose the best split in each node. Random state 0 controls permutation of the features.

\subsubsection{Random Forest}

The Random Forest (RF) comprises of several decision trees which operate individually [45]. The 'Gini index' of each branch is used to find the more likely decision branch to occur. This index calculated by Equation (8):

$$
\text { Gini }=1-\sum_{l=1}^{c}\left(p_{i}\right)^{2}
$$

Here, $c$ represents the total number of class and $p_{i}$ indicated the probability of the $i^{\text {th }}$ class. We used 100 trees in the forest where the quality of split is measured by 'gini'. Internal nodes are split if at least two nodes are there and all the system features are considered in each node.

\subsubsection{Multinomial Naïve Bayes}

Multinomial Naïve Bayes (MNB) is useful to classify discrete features such as document or text classification [46]. MNB follows multinomial distribution and uses a Bayes theorem where variables $V_{1}, V_{2}, \ldots, V_{n}$ of class $C$ are conditionally independent of each other given C [47]. Equations (9) and (10) used MNB for text classification in our dataset:

$$
\begin{aligned}
p(C \mid V) & =\frac{p(V \mid C) p(C)}{p(V)} \\
p\left(C \mid\left(v_{1}, v_{2}, \ldots, v_{n}\right)\right. & =\frac{p\left(v_{1} \mid C\right) p\left(v_{2} \mid C\right) \ldots p\left(v_{n} \mid C\right) p(C)}{p\left(v_{1}\right) p\left(v_{2}\right) \ldots p\left(v_{n}\right)} \\
& =\frac{p(C) \prod_{i=1}^{n} p\left(v_{i} \mid C\right)}{p\left(v_{1}\right) p\left(v_{2}\right) \ldots p\left(v_{n}\right)}
\end{aligned}
$$

Here, $C$ is the class variable and $V=\left(v_{1}, v_{2}, \ldots, v_{n}\right)$ represents the feature vector. We assume that features are conditionally independent. The denominator remains constant for any given input; thus, it can be removed:

$$
C=\operatorname{argmax}_{C} p(C) \prod_{i=1}^{n} p\left(v_{i} \mid C\right)
$$

Equation (10) is used to compute the probability of a given set of inputs for all possible values of class $C$ and pick up the output with maximum probability. Laplace smoothing used and prior probabilities of a class are adjusted according to the data.

\subsection{Prediction}

In this step, the trained classifier models have been used for classification. The test set $T S=\left\{t_{1}, t_{2}, t_{3}, \ldots, t_{x}\right\}$ has $x$ test documents, which will be used to test the classifier model. 
Predicted class $(C)$ is determined by using threshold $\left(T_{h}\right)$ on the predicted probability $(P)$ using Equation (11):

$$
C= \begin{cases}\text { Non-suspicious }\left(C_{n s}\right) & \text { if } P<=T_{h} \\ \operatorname{Suspicious}\left(C_{s}\right) & \text { if } P>T_{h}\end{cases}
$$

The proposed approach classifies suspicious and non-suspicious classes as a binary classification, so sigmoid activation function is used without tweaking the default value of $T_{h}$. It ensured that both train and test documents from the same distribution; otherwise, evaluation will not be accurate.

\section{Experiments}

The goal of the experiments is to analyze the performance of different machine learning classifiers for various feature combinations. We will use several graphical and statistical measures to find out the most suitable model that can perform well for the task of suspicious text classification. Experimentation was carried out in an open-source Google colab platform with Python $==3.6 .9$ and TensorFlow $==2.2 .1$ [48]. Pandas $==1.0 .3$ data frame used for dataset preparation and training and testing purpose, scikit-learn $==0.22 .2$ used. The dataset was partitioned into two independent sets: training and testing. Data are randomly shuffled before partitioning to dispel any bias. The training set is comprised of $80 \%$ of the total data (5600 text documents), and the testing set has $20 \%$ of the total data (1400 text documents). In this section, we subsequently discuss the measures of evaluation and analyze the results of experiments. In addition, we compare the proposed model with existing techniques as well as the human baseline.

\subsection{Measures of Evaluation}

Various statistical and graphical measures are used to calculate the efficiency of the system. The following terminologies have been used for evaluation purposes:

- True Positive $\left(\tau_{p}\right)$ : Texts $\left(t_{i}\right)$ correctly classified as suspicious $\left(C_{s}\right)$.

- False Positive $\left(\Phi_{p}\right)$ : Texts $\left(t_{i}\right)$ incorrectly classified as suspicious $\left(C_{s}\right)$.

- True Negative $\left(\tau_{n}\right)$ : Texts $\left(t_{i}\right)$ correctly classified as non-suspicious $\left(C_{n s}\right)$.

- False Negative $\left(\Phi_{n}\right)$ : Texts $\left(t_{i}\right)$ incorrectly classified as non-suspicious $\left(C_{n s}\right)$.

- Precision: It tells how many of the $t_{i}$ are actually $C_{s}$ among the $t_{i}$ that are classified as $C_{s}$. Precision is calculated by Equation (12):

$$
P=\left(\tau_{p}\right) /\left(\tau_{p}+\Phi_{p}\right)
$$

- Recall: It gives the value of how many text documents $t_{i}$ classified correctly as $C_{s}$ among total suspicious texts. Recall can compute by using Equation (13):

$$
R=\left(\tau_{p}\right) /\left(\tau_{p}+\Phi_{n}\right)
$$

- $\quad f_{1}$-score: This is a useful evaluation metric to decide which classifier to choose among several classifiers. It is calculated by averaging precision and recall, which is done by Equation (14):

$$
f_{1} \text {-score }=(2 * P * R) /(P+R)
$$

As the dataset is balanced, the receiver operating characteristics (ROC) curve is therefore used for the graphical evaluation. The trade-off between the true and false positive rate is summarized by it for different probability thresholds. 


\subsection{Evaluation Results}

We used scikit-learn, a popular machine learning library to implement ML classifiers. Parameters of the classifiers tuned during experimentation. A summary of the parameters used for each classifier is presented in Table 6 .

The 'L2' regularization technique used with 'lbfgs' optimizer in logistic regression. The inverse of the regularization strength set to 1 . We select criterion as 'entropy' and 'gini' for DT and RF, respectively, to measure the quality of a split. Both cases utilize all system features and select the best split at each internal node of DT. We implement RF with 100 decision trees. Each node of the decision branch is divided if it has at least two samples. In MNB, we applied adaptive smoothing and prior probabilities adjusted according to the samples of the class. In the SGD classifier, we selected 'log' loss function and ' 12 ' regularization with the optimal learning rate. Samples were shuffled randomly with a state 0 during training for a maximum of 40 iterations.

Table 6. Summary of the classifier parameters.

\begin{tabular}{|c|c|}
\hline Classifiers & Parameters \\
\hline LR & penalty $={ }^{\prime} l_{2}$ ', C $=1.0$, solver $=$ 'lbfgs', max_iter $=100$ \\
\hline DT & $\begin{array}{c}\text { criterion }=\text { 'entropy' } \text {, splitter }=\text { 'best' } \\
\text { max_features }=\text { n_features, random_state }=0\end{array}$ \\
\hline $\mathrm{RF}$ & $\begin{array}{c}\text { n_estimators }=100, \text { criterion }=\text { 'gini', } \\
\text { min_samples_split }=2, \text { max_features }=\text { n_features }\end{array}$ \\
\hline MNB & alpha $=1.0$, fit_prior $=$ true, class_prior $=$ none \\
\hline SGD & $\begin{array}{c}\text { loss }=\text { 'log' }^{\prime} \text {, penalty }={ }^{\prime} l_{2}{ }^{\prime} \text {, learning_rate }=\text { 'optimal', } \\
\text { max_iter }=40, \text { random_state }=0\end{array}$ \\
\hline
\end{tabular}

\subsubsection{Statistical Evaluation}

The proposed system experimented with five different classification algorithms for BoW and tf-idf feature extraction techniques with n-gram features. The final system evaluated with $\mathrm{F} 1=$ unigram, F2 = bigram, F3 = trigram, F4 = (unigram + bigram $)$ and F5 = (unigram + bigram + trigram $)$ features. Table 7 shows the comparison of performance between the classifiers for a different combination of features. For the BoW FE technique, random forest with an F1 feature outdoes others by acquiring $83.21 \%$ accuracy. There exists a little (0.5-1)\% margin among the classifiers for F1, F2, and F5 features. All of the classifiers obtain the highest accuracy value by employing the F1 feature except DT and SGD. DT performed well with F2 feature, whereas SGD performed for F4 features. All the classifiers showed lower performance with F3 features. SGD achieved the highest precision value of $83.79 \%$, and results showed a minimum difference between precision and recall in SGD.

For the tf-idf FE technique, SGD with an F4 feature obtains the maximum accuracy of $84.57 \%$ where the maximum precision value of $83.78 \%$ achieved for $\mathrm{F} 1$ features. By comparing the results of two feature extraction techniques (i.e., BoW and tf-idf), impressive outcomes have observed in all the evaluation parameters. Almost all the metric values have increased (2-3)\% approximately by adopting the tf-idf feature extraction technique. LR and RF obtained maximum accuracy with F1 features, MNB and SGD gained with F4 feature and DT obtained with F2 features. Thus, in summary, the tf-idf feature extraction and SGD classifier are well suited for our task as it outperforms the BoW technique with other classifiers.

Figure 4 depicts the $f_{1}$-score comparison among the classifiers for the tf-idf feature extraction technique. We observed a tiny margin in the $f_{1}$-score among the classifiers with F1 and F5 features. All classifiers achieved a minimum $f_{1}$-score for the F3 feature except DT. The DT method obtained a minimum value of $78.74 \%$ with the F4 feature. LR and RF got the maximum value of $86.58 \%$ and $86.92 \%$, respectively, for the F1 feature. DT obtained a maximum $f_{1}$-score of $82.81 \%$ while MNB got 
$86.57 \%$. The results revealed that SGD with the F2 feature outperforms all other feature combinations by obtaining an $86.97 \% f_{1}$-score.

Table 7. Performance comparison for different feature combinations where F1, F2, F3, F4, and F5 means unigram, bigram, trigram, a combination of unigram and bigram, and a combination of unigram, bigram, and trigram features, respectively. A, P, and R denotes accuracy, precision, and recall, respectively.

\begin{tabular}{|c|c|c|c|c|c|}
\hline Classifier & FE & Features & A (\%) & P (\%) & R (\%) \\
\hline \multirow{10}{*}{ LR } & \multirow{5}{*}{ BoW } & F1 & 82.28 & 79.46 & 91.91 \\
\hline & & F2 & 81.64 & 77.24 & 94.99 \\
\hline & & F3 & 78.07 & 72.18 & 98.58 \\
\hline & & $\mathrm{F} 4$ & 82.07 & 79.72 & 90.88 \\
\hline & & F5 & 82.21 & 79.57 & 91.52 \\
\hline & \multirow{5}{*}{ tf-idf } & F1 & 84.00 & 81.14 & 92.81 \\
\hline & & F2 & 81.50 & 77.36 & 94.35 \\
\hline & & F3 & 77.85 & 72.22 & 97.81 \\
\hline & & F4 & 83.85 & 80.75 & 93.19 \\
\hline & & F5 & 83.92 & 80.84 & 93.19 \\
\hline \multirow{10}{*}{ DT } & \multirow{5}{*}{ BoW } & F1 & 76.00 & 76.78 & 81.51 \\
\hline & & F2 & 77.78 & 77.14 & 85.36 \\
\hline & & F3 & 74.57 & 69.24 & 97.68 \\
\hline & & F4 & 75.57 & 76.67 & 80.61 \\
\hline & & F5 & 75.50 & 77.38 & 79.07 \\
\hline & \multirow{5}{*}{ tf-idf } & F1 & 77.92 & 78.24 & 83.56 \\
\hline & & $\mathrm{F} 2$ & 79.57 & 77.85 & 88.44 \\
\hline & & F3 & 76.14 & 70.69 & 97.56 \\
\hline & & $\mathrm{F} 4$ & 75.35 & 75.71 & 82.02 \\
\hline & & F5 & 76.71 & 76.93 & 83.05 \\
\hline \multirow{10}{*}{ RF } & \multirow{5}{*}{ BoW } & F1 & 83.21 & 79.43 & 94.22 \\
\hline & & $\mathrm{F} 2$ & 80.50 & 78.68 & 89.08 \\
\hline & & F3 & 76.00 & 70.49 & 97.81 \\
\hline & & F4 & 82.14 & 79.29 & 91.91 \\
\hline & & F5 & 83.20 & 79.82 & 93.45 \\
\hline & \multirow{5}{*}{ tf-idf } & F1 & 83.71 & 78.54 & 97.30 \\
\hline & & F2 & 81.57 & 78.22 & 96.68 \\
\hline & & F3 & 77.92 & 72.16 & 98.20 \\
\hline & & $\mathrm{F} 4$ & 83.21 & 78.14 & 96.91 \\
\hline & & F5 & 83.71 & 78.90 & 96.53 \\
\hline \multirow{10}{*}{ MNB } & \multirow{5}{*}{ BoW } & F1 & 81.57 & 79.10 & 90.88 \\
\hline & & F2 & 79.00 & 74.37 & 94.99 \\
\hline & & F3 & 65.50 & 61.84 & 99.22 \\
\hline & & F4 & 81.14 & 77.77 & 92.55 \\
\hline & & F5 & 81.00 & 77.14 & 93.58 \\
\hline & \multirow{5}{*}{ tf-idf } & F1 & 83.78 & 81.29 & 92.04 \\
\hline & & F2 & 80.35 & 76.25 & 93.96 \\
\hline & & F3 & 73.21 & 67.84 & 98.58 \\
\hline & & F4 & 83.85 & 80.55 & 93.58 \\
\hline & & F5 & 83.50 & 80.17 & 93.45 \\
\hline
\end{tabular}


Table 7. Cont.

\begin{tabular}{cccccc}
\hline Classifier & FE & Features & A (\%) & P (\%) & R (\%) \\
\hline \multirow{6}{*}{ SGD } & F1 & 81.00 & 80.86 & 86.26 \\
& BoW & F2 & 81.00 & 76.58 & 94.86 \\
& & F3 & 78.21 & 72.31 & 98.58 \\
& & F4 & 81.28 & 83.79 & 82.28 \\
& & F5 & 78.57 & 81.30 & 79.84 \\
\hline \multirow{3}{*}{ tf-idf } & F1 & 82.14 & 83.78 & 81.51 \\
& & F2 & 82.00 & 78.06 & 94.09 \\
& & F3 & 78.92 & 73.54 & 97.04 \\
& F4 & $\mathbf{8 4 . 5 7}$ & 82.09 & 92.42 \\
& F5 & 83.92 & 81.04 & 93.53 \\
\hline
\end{tabular}

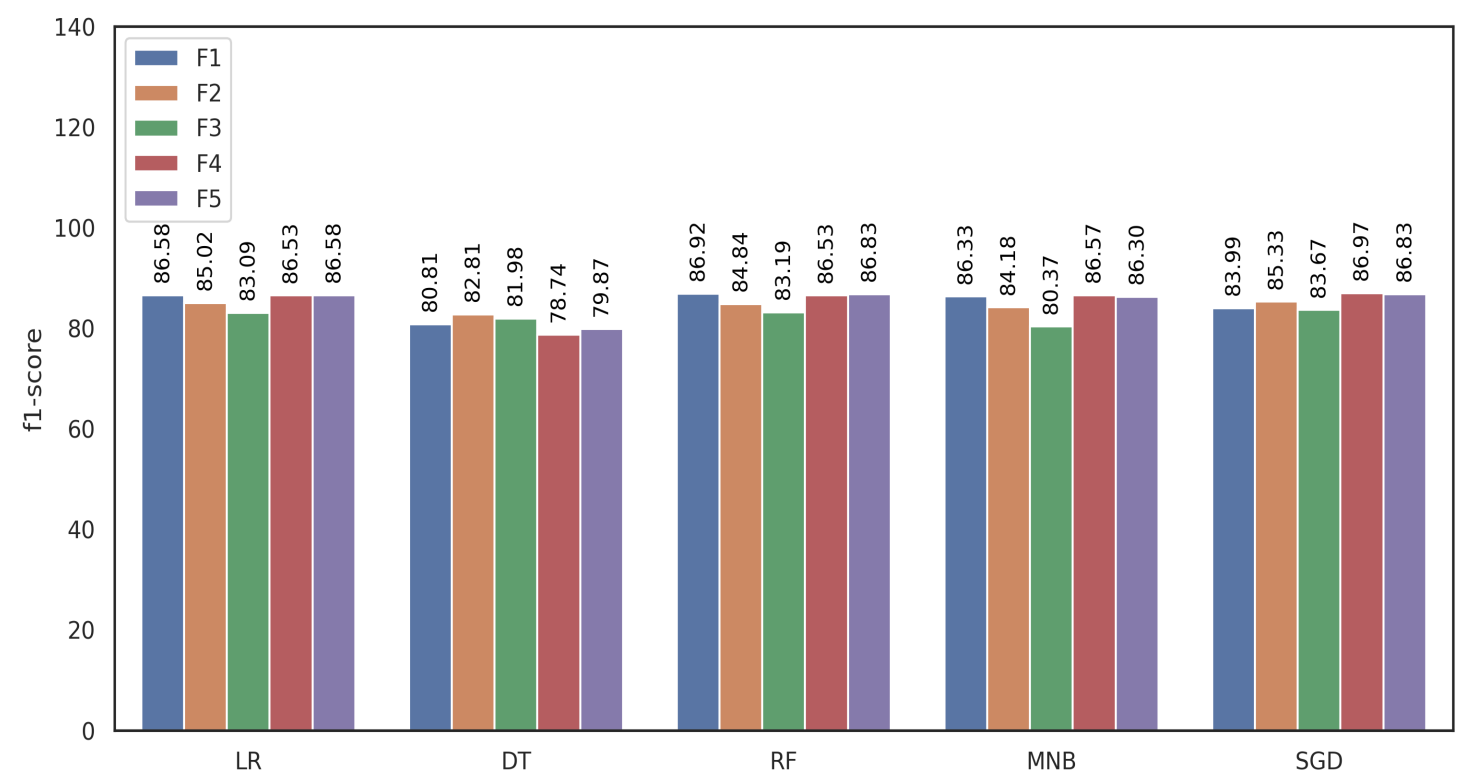

Figure 4. $f_{1}$-score comparison among different ML classifiers with F1, F2, F3, F4, and F5 features for the tf-idf FE technique.

\subsubsection{Graphical Evaluation}

The ROC curve used as a measure of graphical evaluation as each class contains an equal number of texts. Figures 5-9 exhibit the ROC curve analysis of the BoW and the tf-idf feature extraction technique for F1, F2, F3, F4, and F5 features, respectively. For BoW with an F1 feature, logistic regression and random forest both provide the similar AUC values of $87.8 \%$. SGD achieved $87.0 \%$ AUC, which increased by $2.3 \%$ by using the tf-idf FE technique. The AUC value of other algorithms also increased by employing the tf-idf feature extraction technique.

With the F2 feature, LR obtained the maximum AUC value of $84.5 \%$, while, for the F3 feature, SGD achieved the maximum value. In both cases, the tf-idf feature extraction technique was used. With tf-idf and the F4 feature, SGD beats others by getting a maximum AUC of $89.3 \%$. The value of all the classifiers increased except the decision tree where its value decreased by $0.06 \%$. Results with the F5 feature is quite similar to the F1 feature. However, LR outdoes SGD here by a margin of $0.02 \%$. Critical analysis of results brings to the notation that the SGD classifier with the combination of unigram and bigram feature for the tf-idf feature extraction technique achieved the highest value for most of the evaluation parameters compared to others. The performance of the proposed classifier (SGD) was analyzed further by varying the number of training documents to get more insight. 
Figure 10 shows the accuracy versus the number of training examples graph. The analysis reveals that the classification accuracy is increasing with the increased dataset and the tf-idf predominates the BoW with an F2 feature.

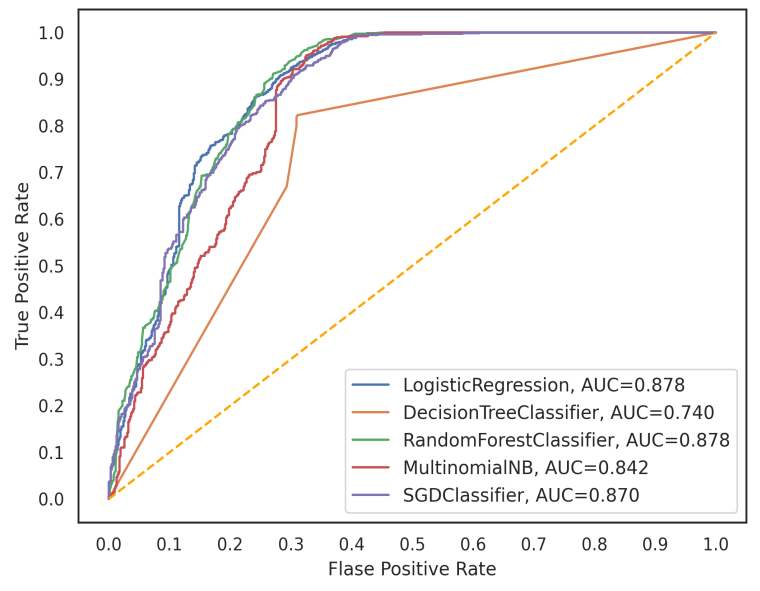

(a) ROC curve for $(\mathrm{BoW}+\mathrm{F} 1)$ features

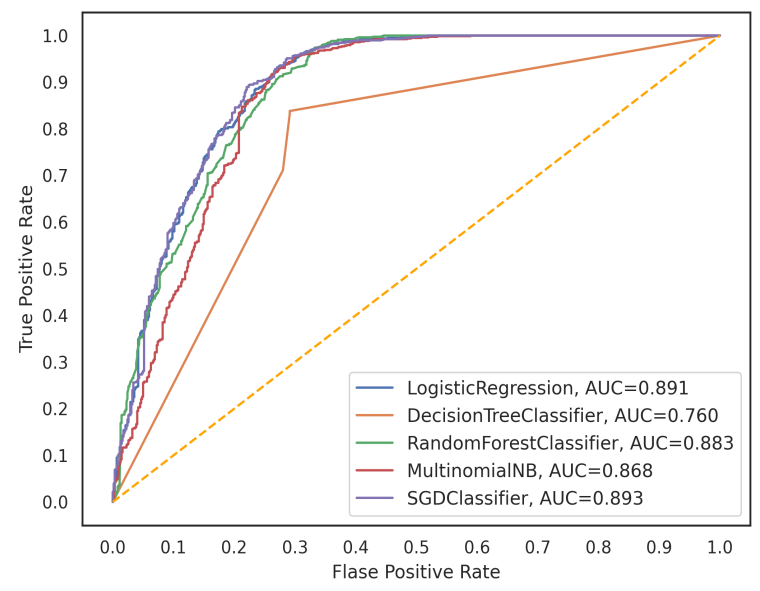

(b) ROC curve for (tf-idf $+\mathrm{F} 1)$ features

Figure 5. ROC curve analysis for the F1 feature where F1 represents the unigram feature.

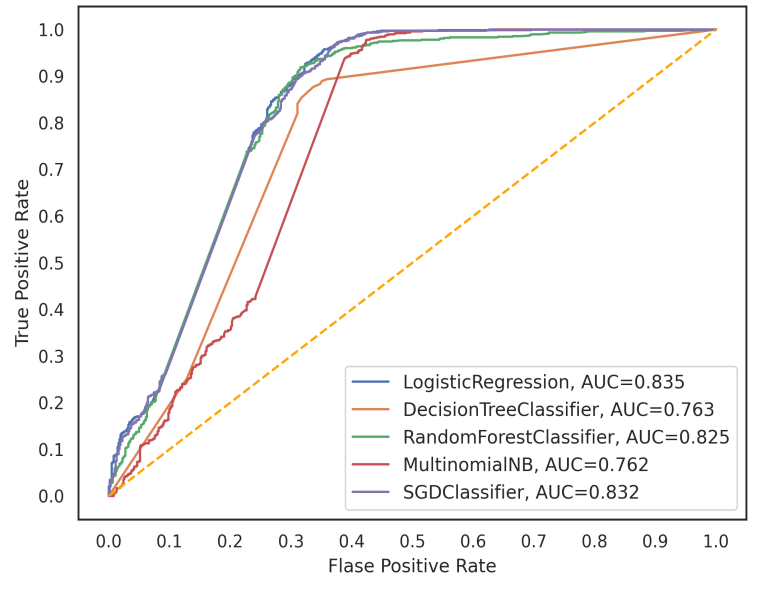

(a) ROC curve for $(\mathrm{BoW}+\mathrm{F} 2)$ features

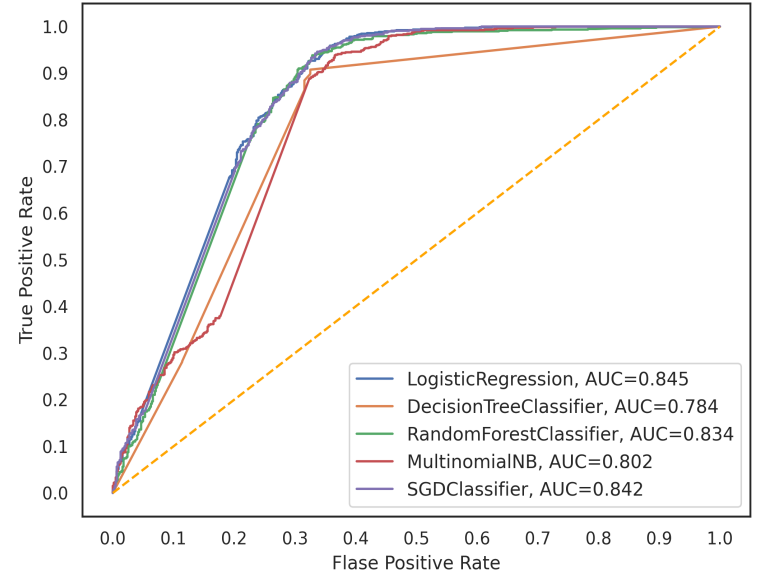

(b) ROC curve for (tf-idf+F2) features

Figure 6. ROC curve analysis for the F2 feature where F2 represents the bigram feature.

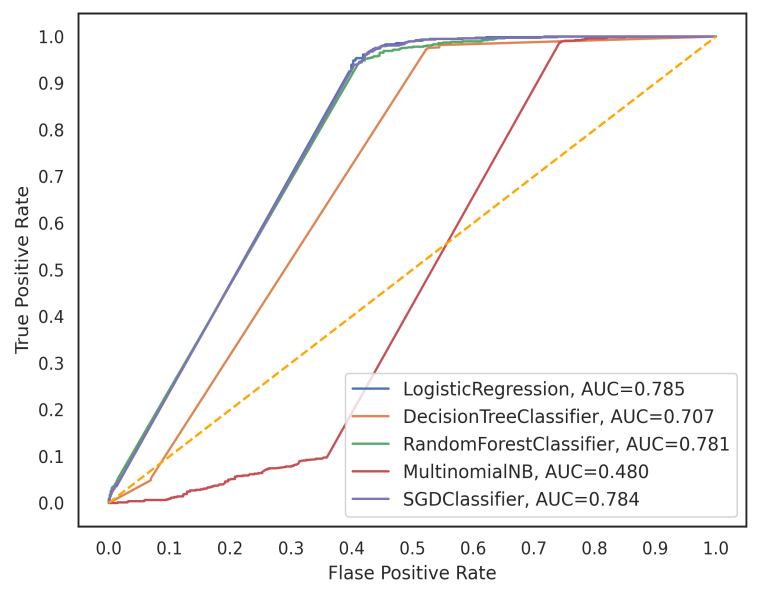

(a) ROC curve for $(\mathrm{BoW}+\mathrm{F} 3)$ features

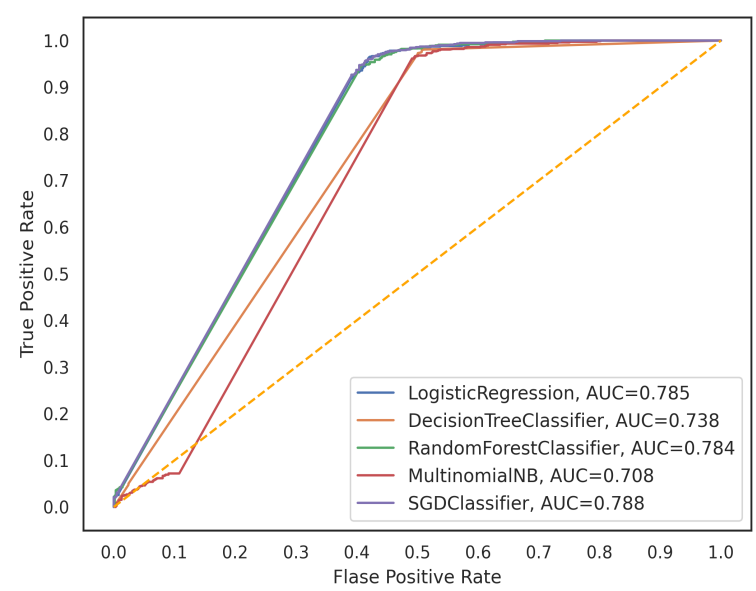

(b) ROC curve for (tf-idf+F3) features

Figure 7. ROC curve analysis for the F3 feature where F3 represents the trigram feature. 


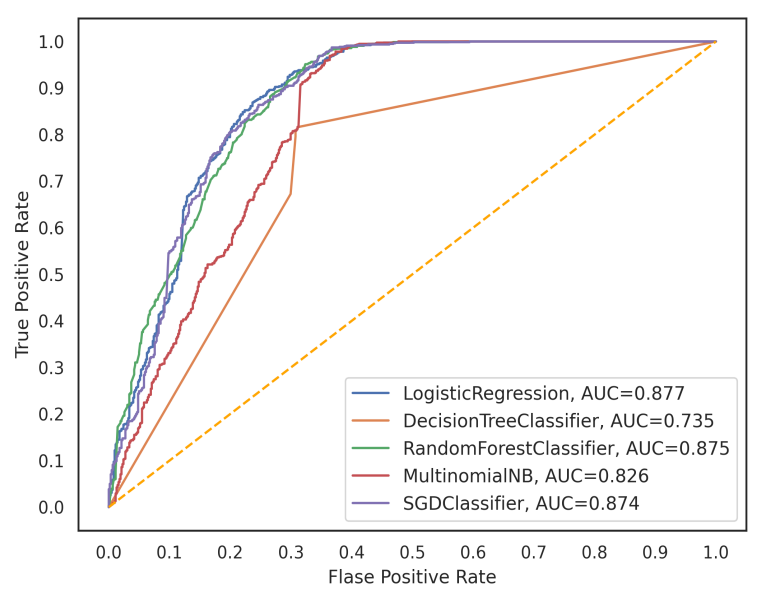

(a) ROC curve for $(\mathrm{BoW}+\mathrm{F} 4)$ features

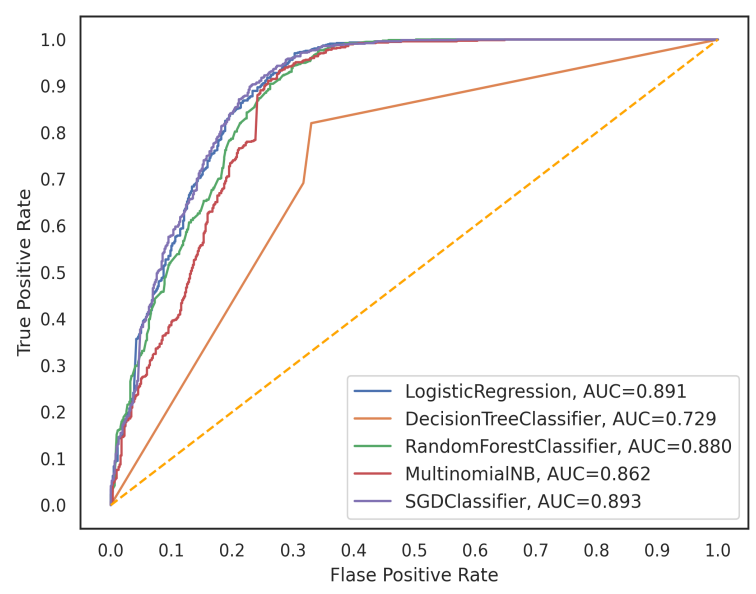

(b) ROC curve for (tf-idf+F4) features

Figure 8. ROC curve analysis for the F4 feature where F4 represents a combination of unigram and bigram features.

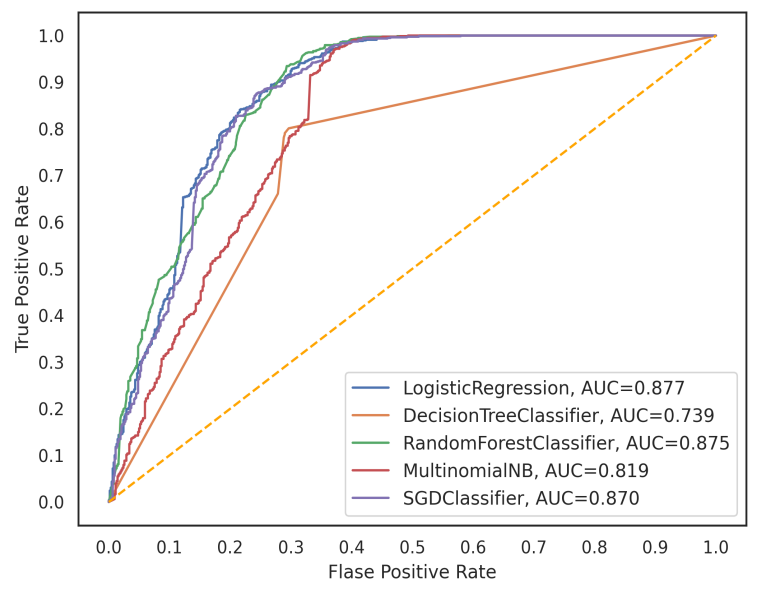

(a) ROC curve for $(\mathrm{BoW}+\mathrm{F} 5)$ features

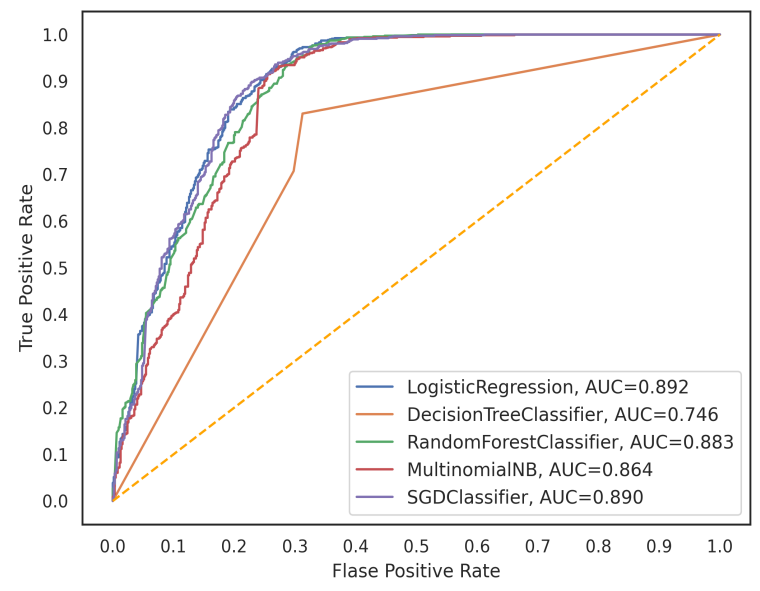

(b) ROC curve for (tf-idf+F5) features

Figure 9. ROC curve analysis for the F5 feature where F5 represents a combination of unigram, bigram, and trigram features.

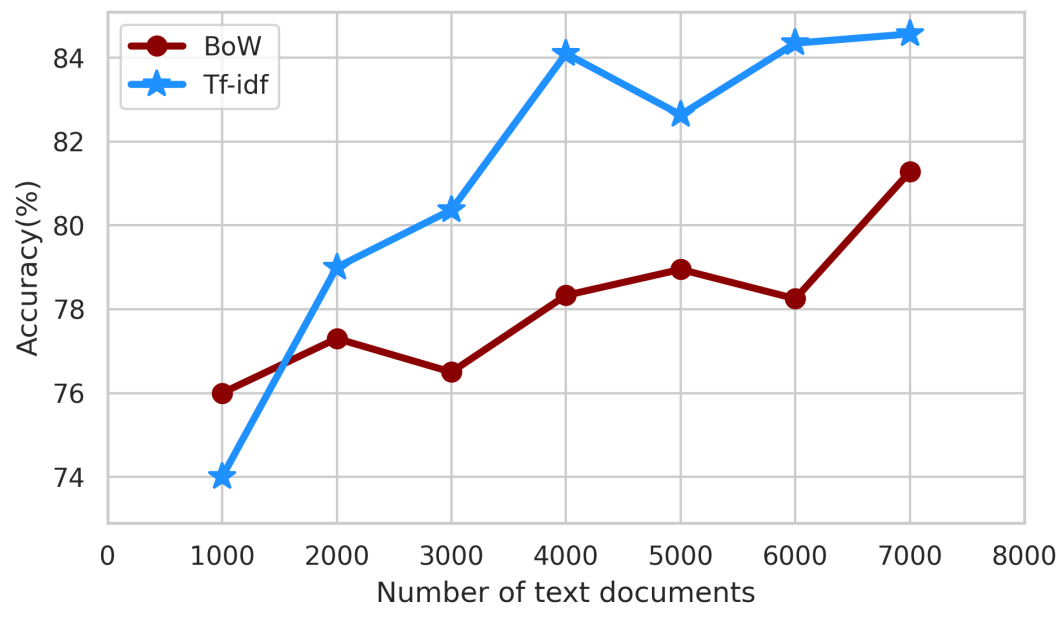

Figure 10. Effects of training set size on accuracy. 


\subsection{Human Baseline vs. ML Techniques}

The performance of the classifiers compared with the human for further investigation. To eliminate the chance of human biases in data labelling and evaluation phases, we have assigned two new experts who manually label the testing texts into one of the predefined categories. Among 1400 test text samples, 621 texts are from non-suspicious $\left(C_{n s}\right)$ class and 779 texts are from suspicious $\left(C_{s}\right)$ class. The accuracy of each class can be computed by the ratio between the number of correctly predicted texts and the total number of texts of that class by using the confusion matrix. Suppose that a system can correctly predict 730 texts among 779 suspicious texts; then, its accuracy in suspicious class will be $93.7 \%$ (730/779). As the tf-idf outperformed the BoW in the previous evaluation, we thus compared the performance of the classifiers only for the tf-idf feature extraction technique with experts. Table 8 exhibits the summary of comparison.

Table 8. Accuracy comparison between experts and classifiers with the tf-idf FE technique.

\begin{tabular}{ccc}
\hline Approach & $C_{n s}$ Accuracy (\%) & $C_{\boldsymbol{s}}$ Accuracy (\%) \\
\hline Expert 1 & 98.71 & 98.58 \\
Expert 2 & 99.19 & 98.33 \\
\hline LR + F1 & 72.94 & 92.81 \\
LR + F2 & 65.37 & 94.35 \\
LR + F3 & 52.81 & 97.81 \\
LR + F4 & 72.14 & 93.19 \\
LR + F5 & 72.30 & 93.19 \\
\hline DT + F1 & 70.85 & 83.56 \\
DT + F2 & 68.43 & 88.44 \\
DT + F3 & 42.44 & 97.56 \\
DT + F4 & 57.69 & 82.02 \\
DT + F5 & 68.76 & 83.05 \\
\hline RF + F1 & 66.66 & 97.30 \\
RF + F2 & 67.63 & 96.68 \\
RF + F3 & 52.49 & 98.20 \\
RF + F4 & 66.02 & 96.91 \\
RF + F5 & 67.63 & 96.53 \\
\hline MNB + F1 & 73.42 & 92.04 \\
MNB + F2 & 63.28 & 93.96 \\
MNB + F3 & 41.38 & 98.58 \\
MNB + F4 & 71.65 & 93.58 \\
MNB + F5 & 71.01 & 93.45 \\
\hline SGD + F1 & 72.30 & 81.51 \\
SGD + F2 & 66.82 & 94.09 \\
SGD + F3 & 56.19 & 97.04 \\
SGD + F4 & 72.46 & 92.42 \\
SGD + F5 & 70.04 & 93.53 \\
\hline & &
\end{tabular}

The experts outperformed the ML classifiers in both classes. Experts can more accurately classify non suspicious texts than suspicious texts. We found approximately $0.5 \%$ accuracy deviation between experts. All of the classifiers done well on the suspicious class and performed very poorly on the non-suspicious class. A significant difference has been observed between human baseline and ML classifiers. All of the classifiers were able to identify suspicious texts more precisely than the non suspicious texts. After manual analysis, we traced the reason behind this disparate behaviour. The maximal portion of the non-suspicious texts was accumulated from the newspaper, and, on average, each text has 72.12 words in it. ML-based classifiers did not consider the semantic meaning of a text which is important for the classification of long texts. Thus, the system could not detect non-suspicious 
texts accurately. For this reason, the false-negative value becomes very high, which causes a drop in the recall, and thus affects the system classification accuracy.

\subsection{Comparison with Existing Techniques}

As far as we aware, no meaningful research has been conducted up to now, which focused solely on suspicious Bengali text classification. In addition to that, no benchmark dataset is available on SBT. Therefore, the proposed work compared with the techniques has already been used on a quite similar task. We implemented existing techniques on our developed dataset to investigate the performance variation of the proposed approach with others. Table 9 shows the comparison in terms of accuracy for the suspicious text classification.

Table 9. Performance comparison after employing existing techniques on our dataset.

\begin{tabular}{cc}
\hline Techniques & Accuracy(\%) on SBTD \\
\hline SVM + BoW [19] & 81.14 \\
LR + unigram + bigram [49] & 82.07 \\
DT + tf-idf [50] & 77.92 \\
Naïve Bayes [51] & 81.77 \\
LR + BoW [30] & 82.28 \\
Our Proposed Technique & 84.57 \\
\hline
\end{tabular}

Naive Bayes [51] and SVM classifier with a BoW [19] feature extraction technique achieved a quite similar accuracy-more than $81 \%$ accuracy on our developed dataset. LR with the combination of unigram and bigram [49] achieved $82.07 \%$ accuracy, whereas LR with the BoW feature extraction technique [30] also achieved similar results (82.28\%). Only $77.92 \%$ accuracy was obtained for DT with the tf-idf feature extraction technique [50], and the proposed method achieved the highest accuracy of $84.57 \%$ among existing approaches. Although the nature of the datasets is different, the result of the comparison indicates that the proposed approach surpasses other existing techniques with the highest accuracy on our developed dataset.

\subsection{Discussion}

After analyzing the experimental results, we can summarize that LR, DT, and RF do well with a unigram feature while MNB and SGD obtained maximum accuracy with unigram and bigram feature combination. In both cases, the tf-idf feature extraction technique is employed. Classifiers performed poorly for trigram features. After comparing BoW and the tf-idf extraction technique, we noticed an allusive rise for the weighted features of the texts. This increase happens because the BoW emphasizes the most frequent words only, while the tf-idf emphasizes the context-related words more. LR, RF, $\mathrm{MNB}$, and SGD performed excellently on every feature combined with a little deviation of $(0.5-0.8) \%$ between them. However, the performance of the decision tree is inferior compared to others due to its limited ability to learn complex rules from texts. The AUC value is another performance measure that indicates the model's capability to make a distinction between classes. SGD obtained the highest value of 0.893 AUC for the tf-idf, and LR and RF achieved the maximum AUC value of 0.878 for the BoW feature extraction. After analysis, the reason behind the superior performance of SGD classifier is pointed out. Here, SGD represents a linear classifier that is already proven as a well-suited model for binary classification like ours [42]. It uses a simple approach of discriminative learning which can find global minima more efficiently, thus resulting in better accuracy. By comparing these ML classifiers in terms of their execution time, no significant difference has been reported. All the classifiers have completed their execution before the 50 s mark.

Since the machine learning-based techniques mainly utilized word-level features, it is difficult to adopt the sentence-level meanings appropriately. The system can not predict the class accurately for this reason. Therefore, to shed light on the tendency for which text is complicated to predict in 
suspicious detection, we analyze the predicted results. Consider an example, (Banglish form: "Sakib al hasan khela pare na take bangladesh cricket team theke ber kore dewa dorkar"). (English translation: Shakib Al Hasan cannot play, he needs to be dropped from the Bangladesh cricket team). This text may excite the fans of Shakib Al Hasan because it conveys the disgraceful message about him. The proposed approach should classify these texts as the suspicious class rather than the non-suspicious class. Thus, the classification discrepancies happen due to the inability to capture the semantic relation between words and sentence-level meanings of the texts. It is always challenging to classify such type of text because these types of texts did not have any words that directly provoke people or pose any threat. The proposed approach encountered a limited number of such texts during the training phase and hence it failed to predict the class correctly. These deficiencies can be dispelled by employing neural network-based architecture with the addition of diverse data in the existing corpus.

Although the result of the proposed SGD based model is quite reasonable compared to the previous approaches, there are scopes to increase the overall accuracy of suspicious Bengali text detection. Firstly, the proposed model did not consider the semantic relation between words in the texts. For this reason, ML-based classifiers show poor accuracy for a non-suspicious class that has long texts. The semantic relationship and corresponding machine learning rule-based model $[52,53]$ could also be effective depending on the data characteristics. Moreover, Deep learning techniques can be used to find the intricate patterns from the texts that will help to comprehend the semantic relation, but it requires a huge amount of data to effectively build the model $[40,54]$. Secondly, the number of classes can be extended by introducing more sub-classes that have suspicious contents such as obscene, religious hatred, sexually explicit, and threats. Finally, to improve the exactness of an intelligent system, it is mandatory to train the model with a diverse and large amount of data. Therefore, a corpus with more texts would help the system to learn more accurately and predict classes more precisely.

\section{Conclusions and Future Research}

In this paper, we have presented a machine learning based model to classify Bengali texts having suspicious contents. We have used different feature extraction techniques with n-gram features in our model. This work also computationally analyzed with a set of ML classification techniques by taking into account the popular BoW and tf-idf feature extraction methods. Moreover, performance of the classifiers is compared with human experts for error analysis. To serve our purpose, a dataset is developed containing 7000 suspicious and non-suspicious text documents. After employing different learning algorithms on this corpus, an SGD classifier and tf-idf feature extraction technique with the combination of unigram and bigram features showed the best performance with $84.57 \%$ accuracy.

In the future, we plan to train the model with a large dataset to increase the overall performance. Sub-domains of suspicious texts will be taken into account to make the dataset more diverse. Furthermore, recurrent learning algorithms can be employed to capture the inherent sequential patterns of long texts.

Author Contributions: Conceptualization, O.S. and M.M.H.; investigation, O.S., M.M.H., A.S.M.K., R.N. and I.H.S.; methodology, O.S. and M.M.H.; software, O.S.; validation, O.S. and M.M.H.; writing—original draft preparation, O.S.; writing-review and editing, M.M.H., A.S.M.K., R.N. and I.H.S. All authors have read and agreed to the published version of the manuscript.

Funding: This research received no external funding.

Conflicts of Interest: The authors declare no conflict of interest.

\section{References}

1. Khangura, A.S.; Dhaliwal, M.S.; Sehgal, M. Identification of Suspicious Activities in Chat Logs using Support Vector Machine and Optimization with Genetic Algorithm. Int. J. Res. Appl. Sci. Eng. Technol. 2017, 5, 145-153.

2. Internet Crime Complaint Center (U.S.), United States, F.B.O.I. 2019 Internet Crime Report. 2020. pp. 1-28. Available online: https: / / www.hsdl.org/?view\&did=833980 (accessed on 22 May 2020) 
3. Bertram, L. Terrorism, the Internet and the Social Media Advantage: Exploring how terrorist organizations exploit aspects of the internet, social media and how these same platforms could be used to counter-violent extremism. J. Deradicalization 2016, 7, 225-252.

4. Mandal, A.K.; Sen, R. Supervised Learning Methods for Bangla Web Document Categorization. Int. J. Artif. Intell. Appl. 2014, 5, 93-105. [CrossRef]

5. Phani, S.; Lahiri, S.; Biswas, A. A Supervised Learning Approach for Authorship Attribution of Bengali Literary Texts. ACM Trans. Asian Low-Resour. Lang. Inf. Process 2017, 16, 1-15. [CrossRef]

6. Facebook. Violence and Incitement. Available online: https://www.facebook.com/communitystandards/ (accessed on 21 April 2019).

7. Fortuna, P.; Nunes, S. A survey on automatic detection of hate speech in text. ACM Comput. Surv. (CSUR) 2018, 51, 1-30. [CrossRef]

8. Understanding Dangerous Speech. Available online: https://dangerousspeech.org/faq/ (accessed on 10 April 2019).

9. Sarker, I.H.; Kayes, A.S.M.; Badsha, S.; Alqahtani, H.; Watters, P.; Ng, A. Cybersecurity data science: An overview from machine learning perspective. J. Big Data 2020, 7, 1-29. [CrossRef]

10. Alami, S.; Elbeqqali, O. Cybercrime profiling: Text mining techniques to detect and predict criminal activities in microblog posts. In Proceedings of the 2015 10th International Conference on Intelligent Systems: Theories and Applications (SITA), Rabat, Morocco, 20-21 October 2015.

11. Hartmann, J.; Huppertz, J.; Schamp, C.; Heitmann, M. Comparing automated text classification methods. Int. J. Res. Mark. 2019, 36, 20-38. [CrossRef]

12. Iskandar, B. Terrorism detection based on sentiment analysis using machine learning. J. Eng. Appl. Sci. 2017, 12, 691-698.

13. Sarker, I.H. A machine learning based robust prediction model for real-life mobile phone data. Internet Things 2019, 5, 180-193. [CrossRef]

14. Johnston, A.H.; Weiss, G.M. Identifying Sunni extremist propaganda with deep learning. In Proceedings of the 2017 IEEE Symposium Series on Computational Intelligence (SSCI), Honolulu, HI, USA, 27 November-1 December 2017.

15. Alami, S.; Beqali, O. Detecting suspicious profiles using text analysis within social media. J. Theor. Appl. Inf. Technol. 2015, 73, 405-410.

16. Jiang, M.; Cui, P.; Faloutsos, C. Suspicious behavior detection: Current trends and future directions. IEEE Intell. Syst. 2016, 31, 31-39. [CrossRef]

17. Vosoughi, S.; Roy, D.; Aral, S. The spread of true and false news online. Science 2018, 359, $1146-1151$. [CrossRef] [PubMed]

18. Davidson, T.; Warmsley, D.; Macy, M.; Weber, I. Automated hate speech detection and the problem of offensive language. In Proceedings of the Eleventh International AAAI Conference on Web and Social Media, Montreal, QC, Canada, 15-18 May 2017.

19. AlGhamdi, M.A.; Khan, M.A. Intelligent Analysis of Arabic Tweets for Detection of Suspicious Messages. Arab. J. Sci. Eng. 2020, 1-12. [CrossRef]

20. Dinakar, K.; Reichart, R.; Lieberman, H. Modeling the detection of textual cyberbullying. In Proceedings of the Fifth International AAAI Conference on Weblogs and Social Media, Barcelona, Catalonia, Spain, 17-21 July 2011.

21. Aulia, N.; Budi, I. Hate Speech Detection on Indonesian Long Text Documents Using Machine Learning Approach. In Proceedings of the 2019 5th International Conference on Computing and Artificial Intelligence, Bali, Indonesia, 19-22 April 2019.

22. Zhang, P.; Gao, Y.; Chen, S. Detect Chinese Cyber Bullying by Analyzing User Behaviors and Language Patterns. In Proceedings of the 2019 3rd International Symposium on Autonomous Systems (ISAS), Shanghai, China, 29-31 May 2019.

23. Hammer, H.L. Detecting threats of violence in online discussions using bigrams of important words. In Proceedings of the 2014 IEEE Joint Intelligence and Security Informatics Conference, The Hague, The Netherlands, 24-26 September 2014.

24. Ishmam, A.M.; Sharmin, S. Hateful Speech Detection in Public Facebook Pages for the Bengali Language. In Proceedings of the 2019 18th IEEE International Conference On Machine Learning And Applications (ICMLA), Boca Raton, FL, USA, 16-19 December 2019. 
25. Emon, E.A.; Rahman, S.; Banarjee, J.; Das, A.K.; Mittra, T. A Deep Learning Approach to Detect Abusive Bengali Text. In Proceedings of the 2019 7th International Conference on Smart Computing \& Communications (ICSCC), Sarawak, Malaysia, 28-30 June 2019.

26. Eshan, S.C.; Hasan, M.S. An application of machine learning to detect abusive bengali text. In Proceedings of the 2017 20th International Conference of Computer and Information Technology (ICCIT), Dhaka, Bangladesh, 22-24 December 2017.

27. Islam, T.; Latif, S.; Ahmed, N. Using Social Networks to Detect Malicious Bangla Text Content. In Proceedings of the 2019 1st International Conference on Advances in Science, Engineering and Robotics Technology (ICASERT), Dhaka, Bangladesh, 3-5 May 2019.

28. Hossain, M.Z.; Rahman, M.A.; Islam, M.S.; Kar, S. BanFakeNews: A Dataset for Detecting Fake News in Bangla. arXiv 2020, arXiv:2004.08789.

29. Chakraborty, P.; Seddiqui, M.H. Threat and Abusive Language Detection on Social Media in Bengali Language. In Proceedings of the 2019 1st International Conference on Advances in Science, Engineering and Robotics Technology (ICASERT), Dhaka, Bangladesh, 3-5 May 2019.

30. Sharif, O.; Hoque, M.M. Automatic Detection of Suspicious Bangla Text Using Logistic Regression. In Proceedings of the International Conference on Intelligent Computing \& Optimization, Koh Samui, Thailand, 3-4 October 2019.

31. Twitter. Hateful Conduct. Available online: https://help.Twitter.com/en/rules-and-policies/Twitterrules/ (accessed on 25 April 2019).

32. Youtube. Harmful or Dangerous Content Policy. Available online: https://support.google.com/youtube/ answer/2801939/ (accessed on 27 April 2019).

33. COE. Hate Speech and Violence. Available online: https://www.coe.int/en/web/european-commissionagainst-racism-and-intolerance/hate-speech-and-violence/ (accessed on 18 April 2019).

34. U.S. Department of Homeland Security. Available online: https://www.dhs.gov/see-something-saysomething/what-suspicious-activity (accessed on 13 April 2019).

35. Dash, N.S.; Ramamoorthy; Naicker, L. Utility E Application of Language Corpora; Springer: Singapore, 2019 ; pp. 17-34.

36. Magatti, D.; Calegari, S.; Ciucci, D.; Stella, F. Automatic labeling of topics. In Proceedings of the 2009 Ninth International Conference on Intelligent Systems Design and Applications, Pisa, Italy, 30 November-2 December 2009.

37. Cohen, J. A coefficient of agreement for nominal scales. Educ. Psychol. Meas. 1960, 20, 37-46. [CrossRef]

38. Tokunaga, T.; Makoto, I. Text categorization based on weighted inverse document frequency. In Special Interest Groups and Information Process Society of Japan (SIG-IPSJ); Citeseer: Tokoyo, Japan, 1994.

39. Fürnkranz, J. A study using n-gram features for text categorization. Austrian Res. Inst. Artif. Intell. 1998, 3, 1-10.

40. Sarker, I.H.; Kayes, A.; Watters, P. Effectiveness analysis of machine learning classification models for predicting personalized context-aware smartphone usage. J. Big Data 2019, 6, 57. [CrossRef]

41. Zhang, T. Solving large scale linear prediction problems using stochastic gradient descent algorithms. In Proceedings of the Twenty-First, International Conference on Machine Learning, New York, NY, USA, 4-8 July 2004.

42. Diab, S. Optimizing stochastic gradient descent in text classification based on fine-tuning hyper-parameters approach. A Case Study on Automatic Classification of Global Terrorist Attacks. arXiv 2019, arXiv:1902.06542.

43. Pranckevičius, T.; Marcinkevičius, V. Application of logistic regression with part-of-the-speech tagging for multi-class text classification. In Proceedings of the 2016 IEEE 4th Workshop on Advances in Information, Electronic and Electrical Engineering (AIEEE), Vilnius, Lithuania, 10-12 November 2016.

44. Pranckevičius, T.; Marcinkevičius, V. Comparison of naive bayes, random forest, decision tree, support vector machines, and logistic regression classifiers for text reviews classification. Balt. J. Mod. Comput. 2017, 5, 221. [CrossRef]

45. Ali, J.; Khan, R.; Ahmad, N.; Maqsood, I. Random forests and decision trees. Int. J. Comput. Sci. Issues (IJCSI) 2012, 9, 272.

46. Le, C.C.; Prasad, P.; Alsadoon, A.; Pham, L.; Elchouemi, A. Text classification: Naïve bayes classifier with sentiment Lexicon. IAENG Int. J. Comput. Sci. 2019, 46, 141-148. 
47. McCallum, A.; Nigam, K. A comparison of event models for naive bayes text classification. In Proceedings of the AAAI-98 Workshop on Learning for Text Categorization, Madison, WI, USA, 26-27 July 1998.

48. Google. Google Colaboratory. Available online: https://colab.research.google.com/notebooks/welcome (accessed on 7 January 2020).

49. Tagami, T.; Ouchi, H.; Asano, H.; Hanawa, K.; Uchiyama, K.; Suzuki, K.; Inui, K.; Komiya, A.; Fujimura, A.; Yanai, H.; et al. Suspicious News Detection Using Micro Blog Text. arXiv 2018, arXiv:1810.11663.

50. Ahmed, H.M.; Bethoon, N.B. Cybercrime: Suspicious Viber Messages Detection Model. Int. J. Sci. Eng. Res. 2017, 8, 1496-1502.

51. Nizamani, S.; Memon, N.; Wiil, U.K.; Karampelas, P. Modeling suspicious email detection using enhanced feature selection. arXiv 2013, arXiv:1312.1971.

52. Sarker, I.H. Context-aware rule learning from smartphone data: survey, challenges and future directions. J. Big Data 2019, 6, 95. [CrossRef]

53. Sarker, I.H.; Kayes, A.S.M. ABC-RuleMiner: User behavioral rule-based machine learning method for context-aware intelligent services. J. Netw. Comput. Appl. 2020, 168, 102762. [CrossRef]

54. Xin, Y.; Kong, L.; Liu, Z.; Chen, Y.; Li, Y.; Zhu, H.; Gao, M.; Hou, H.; Wang, C. Machine learning and deep learning methods for cybersecurity. IEEE Access 2018, 6, 35365-35381. [CrossRef]

Sample Availability: Samples of the compounds are available from the authors.

(C) 2020 by the authors. Licensee MDPI, Basel, Switzerland. This article is an open access article distributed under the terms and conditions of the Creative Commons Attribution (CC BY) license (http://creativecommons.org/licenses/by/4.0/). 